\title{
Blood and Urine Biomarkers Predicting Worsening Kidney Function in Patients with Type 2 Diabetes Post-Acute Coronary Syndrome: An Analysis from the EXAMINE Trial
}

\author{
João Pedro Ferreiraa, ${ }^{a}$ Patrick Rossignol ${ }^{a} \quad$ George Bakris $^{c} \quad$ Cyrus Mehta ${ }^{d}$ \\ William B. White ${ }^{\mathrm{e}}$ Faiez Zannad ${ }^{\mathrm{a}}$ \\ ${ }^{a}$ Centre D'Investigation Clinique-Plurithématique Inserm CIC-P 1433, Inserm U1116, CHRU Nancy Hopitaux de \\ Brabois, F-CRIN INI-CRCT (Cardiovascular and Renal Clinical Trialists), Université de Lorraine, Institut Lorrain Du

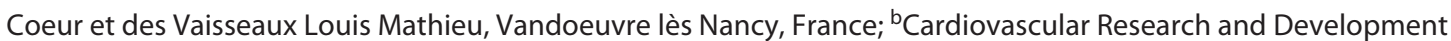 \\ Center, Department of Surgery and Physiology, Faculty of Medicine of the University of Porto, Porto, Portugal; \\ 'Department of Medicine, American Heart Association Comprehensive Hypertension Center, University of Chicago,

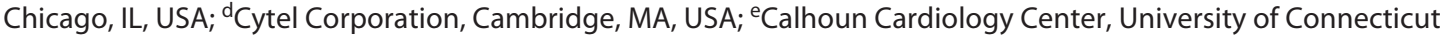 \\ School of Medicine, Farmington, CT, USA
}

\section{Keywords}

Kidney injury molecule $1 \cdot$ Worsening kidney function . Type 2 diabetes · Acute coronary syndromes · Prognosis

\begin{abstract}
Introduction: Worsening kidney function (WKF) is frequent among patients with type 2 diabetes (T2D) and a recent acute coronary syndrome (ACS) and is associated with a poor prognosis. An accurate prediction of WKF is clinically important. Aims: Using data from the Cardiovascular Outcomes Study of Alogliptin in Patients with Type 2 Diabetes and Acute Coronary Syndrome trial including patients with $\mathrm{T} 2 \mathrm{D}$ and a recent ACS, and a large biomarker panel incorporating proteins measured both in blood and urine, we aim to determine those with best performance for WKF prediction. Methods: WKF was defined as a $\geq 40 \%$ estimated glomerular filtration rate (eGFR) drop from baseline, eGFR $<15 \mathrm{~mL} / \mathrm{min}$, or dialysis. Mixed-effects and time-updated Cox models were used. Results: 5,131 patients were included from whom $222(4.3 \%)$ developed at least one WKF episode over a median follow-up of 18 months. Patients who developed WKF were more frequently women, had longer diabetes duration, a more frequent heart failure
\end{abstract}

history, higher anemia prevalence, and impaired kidney function. In multivariable models including all variables (clinical and biomarkers) independently associated with WKF with a $p$ value $\leq 0.0001$, blood kidney injury molecule 1 (KIM-1) was (by far) the variable with strongest WKF association, followed by anemia. KIM-1 alone provided good discrimination for WKF prediction (area under the curve $=0.73$ ). Patients in the high KIM-1-derived risk tertile had a 6.7-fold higher risk of any WKF than patients classified as low risk. In time-updated Cox models, the occurrence of WKF was independently associated with a higher risk of death: adjusted hazard ratio $=4.93(3.06-7.96)$, $p$ value $<0.0001$. Conclusion: Blood KIM- 1 was the biomarker with the strongest association with WKF. The occurrence of WKF was independently associated with a higher risk of subsequent cardiovascular events and mortality.

(c) 2021 S. Karger AG, Basel

\section{Introduction}

Worsening kidney function (WKF) is frequent among patients with type 2 diabetes (T2D) and is associated with a poor prognosis [1]. The risk of WKF is particularly el-
Correspondence to:

João Pedro Ferreira, j.ferreira@chru-nancy.fr 
evated in patients with T2D with a very high cardiovascular risk, such as those with a recent acute coronary syndrome (ACS) or myocardial infarction (MI) $[2,3]$.

Blood and urine biomarkers reflecting kidney damage have been shown to predict WKF and cardiovascular events in patients with and without T2D [4-8]. However, the identified biomarkers vary largely between studies, which poses difficulties in selecting biomarkers for clinical implementation [9].

The use of large biomarker panels incorporating both urinary and blood biomarkers may help in selecting the biomarkers with the best performance in predicting WKF. An accurate identification of patients at higher risk for WKF is important to select those who might experience a greater benefit from therapies that reduce kidney events and improve cardiovascular outcomes in patients with T2D [10-13]. Using data from the Cardiovascular Outcomes Study of Alogliptin in Patients with Type $2 \mathrm{Di}$ abetes and Acute Coronary Syndrome (EXAMINE) trial [14], and a large biomarker panel incorporating proteins measured both in blood and urine, we aim to determine those with best performance for prediction of WKF.

\section{Methods}

\section{Study Design}

Details of the EXAMINE trial design have been previously published $[14,15]$. In short, a total of 5,380 patients with T2D who had a recent ACS were randomly assigned to either alogliptin or placebo. In the overall population, alogliptin significantly reduced glycated hemoglobin without increasing the rates of major adverse cardiovascular events over a median follow-up of 18 months.

The steering committee, consisting of academic members and 3 nonvoting representatives of the sponsor (Takeda Development Center Americas), designed and oversaw the conduct of the trial. An independent data and safety monitoring committee monitored the trial and had access to the unblinded data. For the present analysis, we included 5,131 patients ( $95.4 \%$ of the overall population) who had biomarkers available.

\section{Study Patients}

Patients were eligible for enrollment if they had received a diagnosis of T2D, were receiving antidiabetic therapy (other than a DPP-4 inhibitor or GLP-1 analog), and had an ACS (MI and unstable angina requiring hospitalization) within 15-90 days prior to randomization. Major exclusion criteria were a diagnosis of type 1 diabetes, unstable cardiac disorders (e.g., advanced heart failure $[\mathrm{HF}]$, refractory angina, uncontrolled arrhythmias, critical valvular heart disease, or severe uncontrolled hypertension), and dialysis within 14 days before screening.

Institutional Review Board approval was obtained, and all patients provided informed consent to participate in the trial. The EXAMINE trial is registered with the clinicaltrials.gov number NCT00968708.

\section{End Points}

The primary endpoint in EXAMINE was a composite of death from cardiovascular causes, nonfatal MI, or nonfatal stroke, adjudicated by an independent committee. In the present analysis, we defined WKF as $\%$ of change in estimated glomerular filtration rate (eGFR) at each study visit (months $1,3,6,9,12$, and every 4 months up to month 32 ; after month 32 , eGFR had $>90 \%$ of values missing) relative to the randomization visit. An eGFR drop $\geq 40 \%$ from randomization or $<15 \mathrm{~mL} / \mathrm{min} / 1.73 \mathrm{~m}^{2}$ or progression to dialysis was considered WKF. A WKF that persisted in 2 or more consecutive visits was considered a sustained worsening kidney function. We used eGFR as calculated by the CKD-EPI formula [16]. Additionally, we studied the association of WKF with subsequent occurrence of the study the primary endpoint, and also the occurrence of the composite of HF hospitalization or cardiovascular death, plus all-cause mortality.

\section{Clinical and Biomarker Measurements}

Medical history and a physical examination were all recorded at randomization. A large protein biomarker panel with 92 biomarkers from a wide range of pathophysiological domains was measured (Olink ${ }^{\circledR}$ CVDII panel) at randomization. We selected this panel because it contains known human cardiovascular and inflammatory markers as well as some exploratory human proteins which may have potential as new markers of cardiovascular disease. These proteins are involved in multiple disease processes such as immune/inflammatory response, angiogenesis, coagulation, and cell adhesion. In short, the biomarkers were measured using a high-throughput technique using the Olink Proseek ${ }^{\circledR}$ Multiplex CVDII $96 \times 96$ kit, which simultaneously measures 92 proteins in $1 \mu \mathrm{L}$ of plasma. The kit uses a proximity extension assay technology, where 92 oligonucleotide-labeled antibody probe pairs bind to their respective target present in the sample. The platform provides $\log 2$ normalized protein expression data wherein a high protein value corresponds to a high protein concentration, but not an absolute quantification. The full proteins names from the CVDII panel are described in the online supplementary Table 1 (see www.karger.com/doi/10.1159/000519436 for all online suppl. material). For further details, visit https://www.olink.com/.

In addition to the 92 Olink $^{\circledR}$ biomarkers, high-sensitivity troponin I (troponin) was measured in plasma samples with the ARCHITECT i2000SR analyzer (Abbott Laboratories ${ }^{\circledR}$ ); urinary neutrophil gelatinase-associated lipocalin was measured by enzyme immunoassay (R\&D systems); and urinary kidney injury molecule-1 (uKIM-1) was measured by a microbead ELISA (R\&D systems). Both urinary neutrophil gelatinase-associated lipocalin and uKIM-1 were indexed to urinary creatinine as recommended by the National Institute for Diabetes and Digestive and Kidney Diseases CKD biomarkers consortium [17].

\section{Statistical Analyses}

Baseline characteristics of the patients were compared between those who developed and those who did not develop WKF throughout the follow-up by means of $\chi^{2}$ tests for categorical variables, $t$ tests for continuous normally distributed variables, and Wilcoxon rank-sum tests for continuous non-normally distributed variables. Mixed-effects models were used to test the association between the biomarker levels and the occurrence of WKF (mixedeffects logistic regression) and the change in eGFR slope (mixed-effects linear regression). All mixed-effects models were 
adjusted on age, sex, baseline eGFR, anemia, diabetes duration, smoking, hypertension, prior HF hospitalization, use of diuretics, insulin, metformin, statins, randomized treatment assignment (alogliptin or placebo), study visit, and randomized treatment by study visits interactions; random effects were assigned to patients in panel data with unstructured covariance matrix to allow for correlation between repeated measures. The associations between biomarker levels and WKF (or eGFR slope) were studied in multivariable models fully adjusted on the variables described in the previous step. To select the biomarker(s) with the strongest WKF predictive power while keeping the models parsimonious, we tested each individual biomarker while adjusting for the full list of covariates described in the previous step, and we retained only the biomarkers associated with WKF with a $p$ value of $0.0001(1 / 10,000)$ or less, which is a stricter selection than the conventionally used Bonferroni correction that would have given a $p$ value of 0.0005 or less $(95 / 0.05=0.0005)$. After this "pre-selection" process, all variables/biomarkers with a $p$ value $\leq 0.0001$ were entered in a multivariable stepwise forward selection model with a $p$ value $<0.05$ used to be retained in the model and we computed the area under the curve (AUC) of the model. After the stepwise selection, we then kept the variables with $p$ value $\leq 0.0001$ from the model while comparing the AUC until we reach the simplest and final model without much compromise of the model's accuracy. Afterward, the linear predictor derived from the final model was divided in tertiles, and WKF events were plotted by tertiles of predicted risk. The association of WKF with subsequent cardiovascular outcomes was studied in time-updated Cox models, both crude and adjusted for the variables above described. All statistical analyses were performed with Stata ${ }^{\circledR}$ (StataCorp. 2019. Stata Statistical Software: Release 16.1. College Station, TX: StataCorp LLC).

\section{Results}

\section{Baseline Patients' Characteristics by WKF Status}

Patients who developed at least one WKF episode during the follow-up ( $n=222 ; 4.3 \%)$ were more frequently women (43 vs. $32 \%$ ), had longer diabetes duration (10 vs. 7 years), were more frequently treated with insulin (41 vs. $30 \%$ ), and less frequently treated with metformin (55 vs. $66 \%$ ), smoked less (10 vs. 14\%), had a more frequent history of HF ( 46 vs. $28 \%$ ), higher prevalence of anemia ( 47 vs. $24 \%)$, more frequent kidney function impairment (eGFR <60: 46 vs. $28 \%$ ), higher blood pressure (SBP >140: 26 vs. $20 \%$ ), and were more frequently treated with diuretics (60 vs. $37 \%)$, and less frequently treated with statins ( 85 vs. $91 \%$ ) (Table 1 ).

\section{Associations with WKF and eGFR Slope Changes}

Alogliptin (vs. placebo) had no statistically significant impact on eGFR slope of change during the follow-up: $-1.11(-2.55$ to 0.33$) \mathrm{mL} / \mathrm{min}, p=0.13$ (online suppl. Figure 1). In multivariable models, the strongest clinical variable associated with WKF was anemia: $\beta=1.39(0.96-$
Table 1. Baseline patients' characteristics by WKF status

\begin{tabular}{|c|c|c|c|}
\hline Variable & No WKF & WKF & $p$ \\
\hline$N=5,131$ & 4,909 & 222 & \\
\hline Age, years & $60.9 \pm 9.9$ & $61.8 \pm 10.1$ & 0.19 \\
\hline Age $>65$ yr, $n(\%)$ & $1,735(35.3)$ & $88(39.6)$ & 0.19 \\
\hline Male, $n(\%)$ & $3,359(68.4)$ & $127(57.2)$ & $<0.001$ \\
\hline Duration of T2D, years & $7.0(2.6,13.5)$ & $10.4(4.0,17.4)$ & $<0.001$ \\
\hline HBA1C, $\%$ & $8.0 \pm 1.1$ & $8.0 \pm 1.0$ & 0.66 \\
\hline Insulin, $n(\%)$ & $1,451(29.6)$ & $90(40.5)$ & $<0.001$ \\
\hline Metformin, $n(\%)$ & $3,265(66.5)$ & $122(55.0)$ & $<0.001$ \\
\hline Sulfonylureas, $n(\%)$ & $2287(46.6)$ & $97(43.7)$ & 0.40 \\
\hline Thiazolidinediones, $n$ (\%) & $117(2.4)$ & $9(4.1)$ & 0.12 \\
\hline $\mathrm{BMI}, \mathrm{kg} / \mathrm{m}^{2}$ & $29.5 \pm 5.5$ & $29.8 \pm 6.4$ & 0.37 \\
\hline Race & & & 0.33 \\
\hline White, $n(\%)$ & $3,580(72.9)$ & $160(72.1)$ & \\
\hline Asian, $n(\%)$ & $997(20.3)$ & $41(18.5)$ & \\
\hline Black, $n(\%)$ & $187(3.8)$ & $10(4.5)$ & \\
\hline Other, $n(\%)$ & $145(3.0)$ & $11(5.0)$ & \\
\hline Smoker (current), $n(\%)$ & $683(13.9)$ & $21(9.5)$ & 0.059 \\
\hline MI (history), $n(\%)$ & $4,320(88.0)$ & $191(86.0)$ & 0.38 \\
\hline $\mathrm{HF}$ (history), $n$ (\%) & $1,369(27.9)$ & $103(46.4)$ & $<0.001$ \\
\hline Stroke (history), $n$ (\%) & $344(7.0)$ & $20(9.0)$ & 0.26 \\
\hline PAD (history), $n$ (\%) & $461(9.4)$ & $27(12.2)$ & 0.17 \\
\hline Atrial fibrillation, $n(\%)$ & $343(7.0)$ & $17(7.7)$ & 0.70 \\
\hline Index event Ml, $n(\%)$ & $3,780(77.2)$ & $169(76.5)$ & 0.81 \\
\hline Index event ACS, $n(\%)$ & $1,118(22.8)$ & $52(23.5)$ & \\
\hline Hemoglobin, $\mathrm{g} / \mathrm{dL}$ & $13.5 \pm 1.6$ & $12.6 \pm 1.6$ & $<0.001$ \\
\hline Anemia, $n(\%)$ & $1,184(24.2)$ & $104(46.8)$ & $<0.001$ \\
\hline eGFR, mL/min $/ 1.73 \mathrm{~m}^{2}$ & $71.2 \pm 21.1$ & $63.9 \pm 27.0$ & $<0.001$ \\
\hline eGFR $<60, n(\%)$ & $1,383(28.2)$ & $102(45.9)$ & $<0.001$ \\
\hline Heart rate, bpm & $71.4 \pm 10.8$ & $72.3 \pm 12.1$ & 0.19 \\
\hline $\mathrm{SBP}, \mathrm{mm} \mathrm{Hg}$ & $128.9 \pm 16.6$ & $132.3 \pm 17.5$ & 0.003 \\
\hline $\mathrm{SBP}>140, n(\%)$ & $983(20.0)$ & $58(26.1)$ & 0.027 \\
\hline Antiplatelet agents, $n$ (\%) & $4,773(97.2)$ & $218(98.2)$ & 0.39 \\
\hline Beta-blockers, $n(\%)$ & $4,034(82.2)$ & $183(82.4)$ & 0.92 \\
\hline ACEi or ARB, $n(\%)$ & $4,054(82.6)$ & $179(80.6)$ & 0.45 \\
\hline Calcium channel blockers, $n$ (\%) & $1,084(22.1)$ & $58(26.1)$ & 0.16 \\
\hline Diuretics, $n(\%)$ & $1,792(36.5)$ & $133(59.9)$ & $<0.001$ \\
\hline Statins, $n(\%)$ & $4,462(90.9)$ & $189(85.1)$ & 0.004 \\
\hline Randomized to alogliptin, $n(\%)$ & $2456(50.0)$ & $126(56.8)$ & 0.050 \\
\hline
\end{tabular}

WKF, worsening kidney function (first occurrence); T2D, type 2 diabetes; HBA1C, glycated hemoglobin; BMI, body mass index; MI, myocardial infarction; HF, heart failure; PAD, peripheral artery disease; ACS, acute coronary syndrome; eGFR, estimated glomerular filtration rate; SBP, systolic blood pressure; ACEi or ARB, angiotensin converting enzyme inhibitors or angiotensin receptor blockers.

1.82), $Z=6.4$ (online suppl. Table 2). In biomarkers (adjusted for clinical variables), the one with strongest association with WKF was blood KIM-1: $\beta=0.84(0.66-1.03)$ per $\log 2$ increase, $Z=9.1$; followed by platelet growth factor, $Z=7.1$; and TRAIL receptor $2, Z=6.9$. A total of 18 biomarkers were independently associated with WKF 
Table 2. Multivariable stepwise models predicting WKF

\begin{tabular}{|c|c|c|c|}
\hline Biomarker & Beta coef. $(95 \% \mathrm{Cl})$ & Z-score & $p$ value \\
\hline \multicolumn{4}{|l|}{ Model 1} \\
\hline KIM-1 & $0.50(0.36-0.65)$ & 6.6 & $<0.00001$ \\
\hline Anemia & $0.70(0.36-1.04)$ & 4.0 & 0.00005 \\
\hline VEGFD & $0.76(0.32-1.20)$ & 3.4 & 0.001 \\
\hline IL4RA & $0.50(0.21-0.80)$ & 3.3 & 0.001 \\
\hline uNGAL & $0.11(0.03-0.19)$ & 2.8 & 0.006 \\
\hline SPON2 & $-0.88(-1.64$ to -0.13$)$ & -2.3 & 0.022 \\
\hline$A \cup C=0.78$ & - & - & - \\
\hline \multicolumn{4}{|l|}{ Model 2} \\
\hline KIM-1 & $0.78(0.63-0.92)$ & 10.6 & 0.00001 \\
\hline Anemia & $0.90(0.54-1.26)$ & 4.9 & 0.00001 \\
\hline$A \cup C=0.76$ & - & - & - \\
\hline \multicolumn{4}{|l|}{ Model 3* } \\
\hline KIM-1 & $0.78(0.66-0.89)$ & 12.9 & 0.00001 \\
\hline$A \cup C=0.73$ & - & - & - \\
\hline
\end{tabular}

KIM-1, kidney injury molecule 1; VEGFD, vascular endothelial growth factor-D; IL4RA, interleukin 4 receptor alfa subunit; UNGAL, urinary neutrophil gelatinase-associated lipocalin; SPON2, spondin $2 ; \mathrm{Cl}$, confidence interval; $\mathrm{AUC}$, area under the curve; WKF, worsening kidney function. The correlation coefficient of KIM-1 with the other proteins was not greater than 0.5 . Model 1 shows the results of the stepwise forward model that started with all the variables with a $p$ value $\leq 0.0001$ shown in the online supplementary Tables 2 and 3 (light green); the retained variables with a $p$ value of $<0.05$ to stay in the model are shown in Model 1 of this table; Model 2 shows the results after keeping only the variables with a $p$ value $\leq 0.0001$ in Model 1; Model 3 is a simplest model retaining only KIM-1, the variable with strongest association with WKF. * Anemia alone gave an $\mathrm{AUC}=0.65$.

with a $p$ value $\leq 0.0001$ ( $Z \leq 3.8)$. uKIM-1 was associated with WKF but with a much weaker association than blood KIM-1, $Z=2.9$ for uKIM-1. All biomarkers listed by descending order of strength of association with WKF are listed in the online supplementary Table 3. All the biomarkers associated with WKF were also associated with eGFR slope deterioration (online suppl. Table 4).

In multivariable models including all the variables (clinical and biomarkers) independently associated with WKF with a $p$ value $\leq 0.0001$, blood KIM- 1 was (by far) the variable with strongest association with WKF, followed by anemia. KIM-1 alone provided good discrimination for the prediction of WKF (AUC $=0.73$ ), while anemia alone provided only moderate discrimination and was much lower than KIM-1 alone (AUC $=0.65$ ). Table 2. The urinary albumin-to-creatinine ratio (UACR) variable contains $42 \%(n=2245)$ missing values. As sensitivity analysis, we have adjusted our models to UACR (plus age, sex, and eGFR) in complete cases $(n=2999)$ and after multiple imputation $(n=5,380)$, and KIM- 1 remained the variable with the strongest association with the occurrence of WKF (online suppl. Table 5).

\section{WKF Events by KIM-1 Levels}

The predicted risk of WKF derived from KIM-1 alone was divided in tertiles (low, intermediate/medium, and high risk). Patients in the high-risk tertile had a 6.7-fold higher risk of any WKF than patients classified as low risk, and the risk of sustained worsening kidney function was 12.5-fold higher in high-risk than low-risk patients (Table 3 and Figure 1).

\section{Events after WKF}

In time-updated Cox models, the occurrence of WKF was independently associated with a higher risk of experiencing a subsequent primary outcome event: adjusted hazard ratio $(\mathrm{HR})=4.25(2.83-6.39)$; cardiovascular death or HF hospitalization: adjusted HR $=6.19$ (4.099.36); and all-cause mortality: adjusted $\mathrm{HR}=4.93$ (3.067.96) (all $p<0.0001)$ (Figure 2).

\section{Discussion}

The present study shows that in more than 5,000 patients with T2D and a recent ACS in whom 95 biomarkers (in both urine and blood) had been measured at baseline, blood KIM-1 was the biomarker with the strongest association with WKF. The occurrence of WKF was independently associated with a higher risk of subsequent cardiovascular events and mortality.

Identifying patients more likely to develop kidney dysfunction is important both for clinical practice and research. To date, the prediction of WKF is often performed by detecting rapid rises in serum creatinine or rapid decreases in eGFR, which is far from ideal, because it frequently represents overt WKF resulting in a late diagnosis, often at advanced stages of kidney injury where therapeutic options are limited and the prognosis is poor [18]. Therefore, more important is predicting the occurrence of WKF before its onset, so that preventive strategies can be implemented [19]. In this regard, several molecules, measured both in blood and urine, have been identified as potential markers for early detection of kidney damage before rises in serum creatinine or eGFR decline [20-23]. However, these markers are not routinely used in clinical practice nor for patient selection in clinical trials, due to limitations in accuracy and reproducibility $[3,6]$. 
Table 3. WKF events by tertiles of risk predicted from blood KIM-1 levels

\begin{tabular}{lllll}
\hline KIM-1 predicted risk & Low & Intermediate & High & Total \\
\hline \multicolumn{1}{l}{ Total WKF (first and recurrent) } & & & \\
No WKF & 11,455 & 11,471 & 11,217 & 34,143 \\
WKF & $44(0.4)$ & $58(0.5)$ & $216(1.9)$ & 318 \\
OR $(95 \%$ CI) & Ref & $1.40(0.83-2.36)$ & $6.68(4.15-10.76)$ & - \\
AUC $=0.73$ & - & - & - & - \\
\hline First WKF & & & & \\
No WKF & 1,675 & 1,662 & 1,572 & 4,909 \\
WKF & $36(2.1)$ & $48(2.8)$ & $138(8.1)$ & 222 \\
HR $(95 \%$ CI) & Ref & $1.37(0.89-2.11)$ & $4.2(2.91-6.06)$ & - \\
C-index $=0.69$ & - & - & - & - \\
\hline sWKF & & & & 5,071 \\
No WKF & 1,707 & 1,700 & 1,644 & 60 \\
WKF & $4(0.2)$ & $10(0.6)$ & $46(2.7)$ & - \\
HR $(95 \%$ CI) & Ref & $2.55(0.80-8.13)$ & $12.46(4.48-34.60)$ & - \\
Cindex $=0.80$ & - & - & - & \\
\hline
\end{tabular}

WKF, worsening kidney function; sWKF, sustained worsening kidney function; KIM-1, kidney injury molecule 1; OR, odds ratio; $\mathrm{HR}$, hazard ratio; $95 \% \mathrm{Cl}, 95 \%$ confidence interval; AUC, area under the curve. Low, intermediate, and high represent the tertiles of predicted risk from KIM-1 levels. KIM-1 predicted the occurrence of WKF in an incremental manner, regardless of the WKF definition.

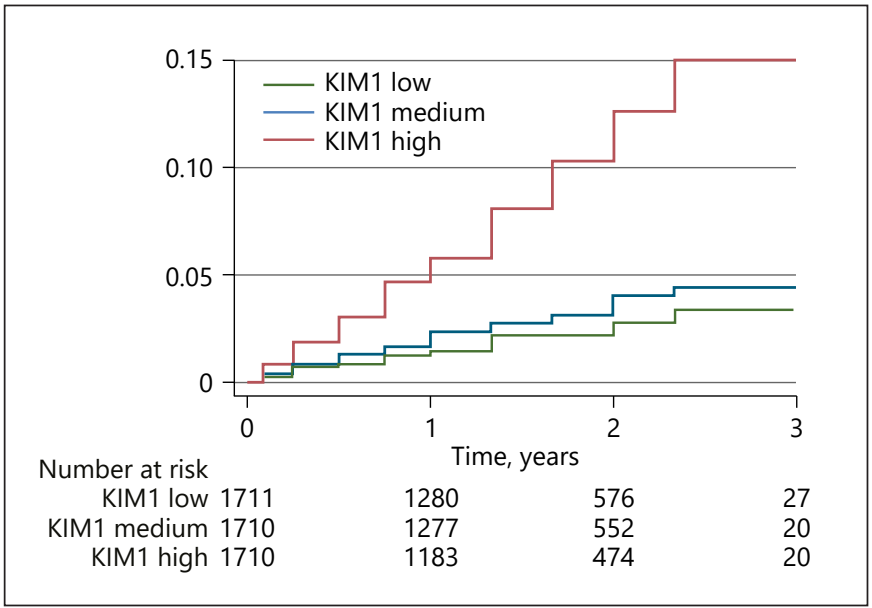

Fig. 1. WKF events by tertiles of risk predicted from blood KIM-1 levels. Caption: Higher KIM-1 levels predict WKF events defined by a deterioration in eGFR $\geq 40 \%$ from baseline. WKF, worsening kidney function; KIM-1, kidney injury molecule 1; eGFR, estimated glomerular filtration rate.

In our study, blood KIM-1 was the biomarker with the strongest association with WKF and was also strongly associated with eGFR slope deterioration over time. KIM-1 is upregulated in the proximal tubular cells following kidney injury [24]. The disruption of renal endothelial cells,

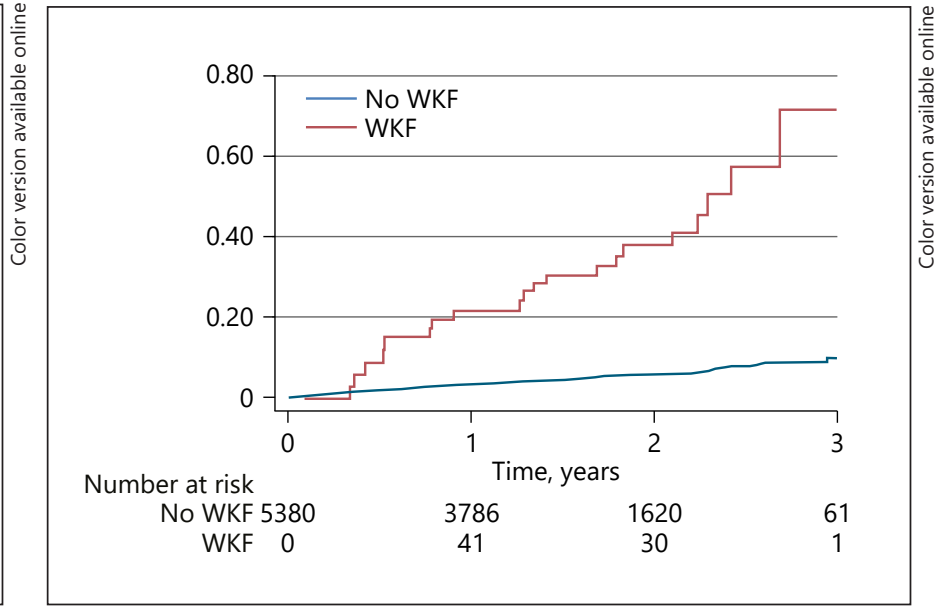

Fig. 2. Death after a WKF event. WKF defined by a deterioration in eGFR $\geq 40 \%$ from baseline. Caption: crude HR $(95 \% \mathrm{CI})=8.05$ (5.08-12.76), $p<0.0001$. WKF, worsening kidney function; eGFR, estimated glomerular filtration rate; $\mathrm{HR}$, hazard ratio; CI, confidence interval.

with loss of cellular adhesion junctions and disruption of extracellular matrix, facilitates KIM-1 movement into the circulation and its blood detection, beyond its urinary excretion. In fact, raised levels of blood KIM-1 may represent a more severe kidney injury due to the disruption of 
the endothelial barrier [25]. Higher levels of blood KIM-1 have been shown to predict acute and chronic kidney injury in patients with type 1 diabetes $[4,26]$, long-term deterioration of kidney function in the general population [27], and poorer renal function in HF [28]. In a study including patients with type 1 diabetes without proteinuria in whom both blood and uKIM-1 were measured, blood KIM-1 (but not uKIM-1) was the biomarker more strongly associated with kidney function decline [26]. In a previous analysis of the EXAMINE trial studying uKIM1 and NGAL not measuring blood biomarkers, neither uKIM-1 nor urinary NGAL improved the prediction of eGFR decline [29]. These findings were concordant with the present analysis, where both blood and UKIM-1 were measured, but blood KIM-1 had a stronger association with WKF and renal function "slope" decline than uKIM1, urinary NGAL, or any other measured biomarker. Our results suggest that damage to proximal tubules with disruption of the endothelial barrier plays an independent role in the development and progression of kidney dysfunction.

Important clinical and research implications can be drawn from these findings. Due to extensive replication and strong prediction capacity, blood KIM-1 could be implemented for the prediction of kidney function decline in the clinical setting, allowing to individualize preventive strategies to patients at higher risk of kidney dysfunction progression. These preventive strategies might include treatment with sodium glucose co-transporter $1 / 2$ inhibitors and finerenone that have been shown to reduce WKF and prevent kidney function decline in patients with and without diabetes [10-13, 30,31]. Interestingly, empagliflozin has been shown to reduce the urinary levels of KIM-1, suggesting that empagliflozin may reduce tubular injury and improve the function of the proximal tubule [32].

Blood KIM-1 can also be used in clinical trials for risk enrichment of kidney outcomes, allowing to increase the number of events and the study power, tailored to patients who might benefit the most from the treatment being studied [33]. Furthermore, along with brain natriuretic peptides and troponin, that strongly predict cardiovascular events [34], the addition of KIM-1 for WKF prediction may allow an optimal risk assessment of cardio-kidney endpoints [33].

\section{Limitations}

Some limitations should be acknowledged in this study. This is a post hoc analysis of a randomized controlled trial subject to biases inherent to observational data. Blood KIM-1 and all the Olink ${ }^{\circledR}$ biomarkers represent normalized concentrations and cannot be translated into cut points ready for clinical use. Further studies using other laboratorial techniques for measuring blood KIM-1 (e.g., ELISA) are warranted to validate our findings. Despite extensive replication of blood KIM-1 as a biomarker strongly associated with WKF in several populations, further data are needed for patients with T2D and an ACS, where our study is unique. UACR has $42 \%$ of missing values in EXAMINE; still in sensitivity analysis adjusting for UACR (plus age, sex, and eGFR), KIM1 remained the variable with strongest association with WKF.

\section{Conclusions}

In patients with $\mathrm{T} 2 \mathrm{D}$ and a recent ACS, blood KIM-1 was the biomarker with the strongest association with WKF. The occurrence of WKF was independently associated with a higher risk of subsequent cardiovascular events and mortality. Further studies are needed to confirm these hypothesis generating findings and assess whether KIM-1 could be implemented for early prediction of kidney outcomes in the clinical setting.

\section{Acknowledgments}

Cardiovascular Outcomes Study of Alogliptin in Patients with Type 2 Diabetes and Acute Coronary Syndrome (EXAMINE) is a clinical trial sponsored by Takeda Global Research and Development Center, Inc., Deerfield, IL. The authors are solely responsible for the design of the study, all study analyses, and the drafting and editing of the manuscript and its final contents. Clinical trials identifier: Clinicaltrials.gov-NCT00968708.

\section{Statement of Ethics}

The appropriate national and institutional regulatory authorities and ethics committees approved the study design, and all participants provided written informed consent.

\section{Conflict of Interest Statement}

Dr Ferreira is a consultant for Boehringer-Ingelheim and receives research support from AstraZeneca and Novartis. Dr Rossignol reports personal fees from Ablative Solutions, AstraZeneca, Bayer, Boehringer-Ingelheim, Corvidia, CVRx, Fresenius, G3P (stocks), Grunenthal, Idorsia, KBP, Novartis, Novo Nordisk, Relypsa, Sanofi, Sequana Medical, Servier, Stealth Peptides, Vifor, Vifor Fresenius Medical Care Renal Pharma, and Cofounder: CardioRenal, a company developing sensors for the home monitoring of 
potassium and creatinine. Dr Zannad reports personal fees from Boehringer-Ingelheim during the conduct of the study; personal fees from Janssen, Novartis, Boston Scientific, Amgen, CVRx, AstraZeneca, Vifor Fresenius, Cardior, Cereno pharmaceutical, Applied Therapeutics, Merck, Bayer, and Cellprothera outside the submitted work; and other support from CVCT and CardioRenal, outside the submitted work. Dr Bakris has received personal fees from Takeda Development Center, is a consultant for Merck, Relypsa, and is on the steering committee for international renal/CV outcomes trials for Janssen, Bayer, and Vascular Dynamics. Dr White has received research support from the National Institute of Aging (NIH) and personal fees from Takeda Development Center (Deerfield, IL, USA) during the conduct of the EXAMINE trial until 2016 (Steering Committee Chair). All the other authors reported not having potential conflicts of interest relevant to this article.

\section{Funding Sources}

The Cardiovascular Outcomes Study of Alogliptin in Patients with Type 2 Diabetes and Acute Coronary Syndrome (EXAMINE) study was funded by Takeda, but the present analysis did not receive funding.

J.P.F., P.R., and F.Z. are supported by a public grant overseen by the French National Research Agency as part of the second "Investissements d'Avenir" programme (ANR-15-RHU-0004). All the other authors have nothing to disclose with regard to this manuscript.

\section{References}

1 Ritz E, Orth SR. Nephropathy in patients with type 2 diabetes mellitus. N Engl J Med. 1999 Oct 7;341(15):1127-33.

2 Potier L, Roussel R, Zeller M, Schiele F, Puymirat E, Simon T, et al. Chronic kidney disease, diabetes, and risk of mortality after acute myocardial infarction: insight from the FAST-MI program. Diabetes Care. 2020;43: e43-4.

3 Legrand M, Rossignol P. Cardiovascular Consequences of Acute Kidney Injury. N Engl J Med. 2020 Jun 4;382(23):2238-47.

4 Sabbisetti VS, Waikar SS, Antoine DJ, Smiles A, Wang C, Ravisankar A, et al. Blood kidney injury molecule- 1 is a biomarker of acute and chronic kidney injury and predicts progression to ESRD in type I diabetes. J Am Soc Nephrol. 2014 Oct;25(10):2177-86.

5 Zhang WR, Craven TE, Malhotra R, Cheung AK, Chonchol M, Drawz P, et al. Kidney damage biomarkers and incident chronic Kidney disease during blood pressure reduction: a case-control study. Ann Intern Med. 2018 Nov 6;169(9):610-8.

6 Ronco C, Bellomo R, Kellum JA. Acute kidney injury. Lancet. 2019 Nov 23;394(10212): 1949-64.

7 Coca SG, Parikh CR. Urinary biomarkers for acute kidney injury: perspectives on translation. Clin J Am Soc Nephrol. 2008 Mar;3(2): 481-90.

\section{Author Contributions}

J.P.F. performed statistical analysis and drafted the manuscript and its subsequent revisions. All authors provided critical input to the manuscript.

J.P.F. wrote the manuscript, performed the statistical analysis, and made critical revisions; C.M. contributed to the oversight of the EXAMINE trial, as a steering committee member and to discussion and reviewed/edited the manuscript including the revision of the statistical methods and analysis; G.B. contributed to the oversight of the EXAMINE trial, as a steering committee member and reviewed/edited the manuscript; P.R. reviewed/edited the manuscript; W.B.W. contributed to the oversight of the EXAMINE trial, as the principal investigator of the trial, and reviewed/ edited the manuscript; F.Z. contributed to the oversight of the EXAMINE trial, as a steering committee member, discussion, and reviewed/edited the manuscript. Dr. Joao Pedro Ferreira is the guarantor of this work and, as such, had full access to all the data in the study and takes responsibility for the integrity of the data and the accuracy of the data analysis.

\section{Data Availability Statement}

The data and materials may be available upon reasonable request.
8 Vaduganathan M, White WB, Charytan DM, Morrow DA, Liu Y, Zannad F, et al. Relation of serum and urine renal biomarkers to cardiovascular risk in patients with type 2 diabetes mellitus and recent acute coronary syndromes (from the EXAMINE trial). Am J Cardiol. 2019 Feb 1;123(3):382-91.

9 Ostermann M, Zarbock A, Goldstein S, Kashani K, Macedo E, Murugan R, et al. Recommendations on acute kidney injury biomarkers from the acute disease quality initiative consensus conference: a consensus statement. JAMA Netw Open. 2020 Oct 1;3(10): e2019209.

10 Zelniker TA, Wiviott SD, Raz I, Im K, Goodrich EL, Bonaca MP, et al. SGLT2 inhibitors for primary and secondary prevention of cardiovascular and renal outcomes in type 2 diabetes: a systematic review and meta-analysis of cardiovascular outcome trials. Lancet. 2019 Jan 5;393(10166):31-9.

11 Bakris GL, Agarwal R, Anker SD, Pitt B, Ruilope LM, Rossing P, et al. Effect of finerenone on chronic kidney disease outcomes in type 2 diabetes. N Engl J Med. 2020 Dec 3; 383(23):2219-29.

12 Bhatt DL, Szarek M, Pitt B, Cannon CP, Leiter LA, McGuire DK, et al. Sotagliflozin in Patients with Diabetes and Chronic Kidney Disease. N Engl J Med. 2021 Jan 14;384(2):12939.
13 Filippatos G, Anker SD, Agarwal R, Pitt B, Ruilope LM, Rossing P, et al. Finerenone and cardiovascular outcomes in patients with chronic kidney disease and type 2 diabetes. Circulation. 2021 Feb 9;143(6):540-52.

14 White WB, Cannon CP, Heller SR, Nissen SE, Bergenstal RM, Bakris GL, et al. Alogliptin after acute coronary syndrome in patients with type 2 diabetes. N Engl J Med. 2013 Oct 3; 369(14):1327-35.

15 White WB, Bakris GL, Bergenstal RM, Cannon CP, Cushman WC, Fleck P, et al. EXamination of cArdiovascular outcoMes with alogliptIN versus standard of carE in patients with type 2 diabetes mellitus and acute coronary syndrome (EXAMINE): a cardiovascular safety study of the dipeptidyl peptidase 4 inhibitor alogliptin in patients with type 2 diabetes with acute coronary syndrome. Am Heart J. 2011 Oct;162(4):620-e1.

16 Ferreira JP, Girerd N, Pellicori P, Duarte K, Girerd S, Pfeffer MA, et al. Renal function estimation and Cockroft-Gault formulas for predicting cardiovascular mortality in population-based, cardiovascular risk, heart failure and post-myocardial infarction cohorts: the heart 'OMics' in AGEing (HOMAGE) and the high-risk myocardial infarction database initiatives. BMC Med. 2016 Nov 10; 14(1):181. 
17 Fufaa GD, Weil EJ, Nelson RG, Hanson RL, Bonventre JV, Sabbisetti V, et al. Association of urinary KIM-1, L-FABP, NAG and NGAL with incident end-stage renal disease and mortality in American Indians with type 2 diabetes mellitus. Diabetologia. 2015 Jan;58(1): 188-98.

18 Singbartl K, Bishop JV, Wen X, Murugan R, Chandra S, Filippi MD, et al. Differential effects of kidney-lung cross-talk during acute kidney injury and bacterial pneumonia. Kidney Int. 2011 Sep;80(6):633-44.

19 Chawla LS, Bellomo R, Bihorac A, Goldstein SL, Siew ED, Bagshaw SM, et al. Acute kidney disease and renal recovery: consensus report of the acute disease quality initiative (ADQI) 16 workgroup. Nat Rev Nephrol. 2017 Apr; 13(4):241-57.

20 Mori K, Lee HT, Rapoport D, Drexler IR, Foster K, Yang J, et al. Endocytic delivery of lipocalin-siderophore-iron complex rescues the kidney from ischemia-reperfusion injury. J Clin Invest. 2005 Mar;115(3):610-21.

21 Ismail OZ, Zhang X, Wei J, Haig A, Denker $\mathrm{BM}$, Suri RS, et al. Kidney injury molecule-1 protects against $\mathrm{Ga} 12$ activation and tissue damage in renal ischemia-reperfusion injury. Am J Pathol. 2015 May;185(5):1207-15.

22 Parikh CR, Thiessen-Philbrook H, Garg AX, Kadiyala D, Shlipak MG, Koyner JL, et al. Performance of kidney injury molecule-1 and liver fatty acid-binding protein and combined biomarkers of AKI after cardiac surgery. Clin J Am Soc Nephrol. 2013 Jul;8(7):1079-88.
23 Kashani K, Al-Khafaji A, Ardiles T, Artigas A, Bagshaw SM, Bell M, et al. Discovery and validation of cell cycle arrest biomarkers in human acute kidney injury. Crit Care. $2013 \mathrm{Feb}$ 6;17(1):R25.

24 Ichimura T, Bonventre JV, Bailly V, Wei H, Hession CA, Cate RL, et al. Kidney injury molecule-1 (KIM-1), a putative epithelial cell adhesion molecule containing a novel immunoglobulin domain, is up-regulated in renal cells after injury. J Biol Chem. 1998 Feb 13;273(7):4135-42.

25 Sutton TA, Fisher CJ, Molitoris BA. Microvascular endothelial injury and dysfunction during ischemic acute renal failure. Kidney Int. 2002 Nov;62(5):1539-49.

26 Nowak N, Skupien J, Niewczas MA, Yamanouchi M, Major M, Croall S, et al. Increased plasma kidney injury molecule-1 suggests early progressive renal decline in non-proteinuric patients with type 1 diabetes. Kidney Int. 2016 Feb;89(2):459-67.

27 Schulz CA, Engström G, Nilsson J, Almgren P, Petkovic M, Christensson A, et al. Plasma kidney injury molecule-1 (p-KIM-1) levels and deterioration of kidney function over 16 years. Nephrol Dial Transpl. 2020 Feb 1; 35(2):265-73

28 Emmens JE, Ter Maaten JM, Matsue Y, Metra M, O’Connor CM, Ponikowski P, et al. Plasma kidney injury molecule- 1 in heart failure: renal mechanisms and clinical outcome. Eur J Heart Fail. 2016 Jun;18(6):641-9.

29 Garlo KG, White WB, Bakris GL, Zannad F, Wilson CA, Kupfer S, et al. Kidney biomark- ers and decline in eGFR in patients with type 2 diabetes. Clin J Am Soc Nephrol. 2018 Mar 7;13(3):398-405.

30 Zannad F, Ferreira JP, Pocock SJ, Anker SD, Butler J, Filippatos G, et al. SGLT2 inhibitors in patients with heart failure with reduced ejection fraction: a meta-analysis of the EMPEROR-Reduced and DAPA-HF trials. Lancet. 2020 Sep 19;396(10254):819-29.

31 Ferreira JP, Oliveira AC, Saraiva FA, VasquesNóvoa F, Leite-Moreira A. Sodium-glucose co-transporter inhibitors in insulin-treated diabetes: a meta-analysis. Eur J Endocrinol. 2021 May 4;184(6):783-90.

32 Opingari E, Verma S, Connelly KA, Mazer $\mathrm{CD}$, Teoh H, Quan A, et al. The impact of empagliflozin on kidney injury molecule-1: a subanalysis of the effects of empagliflozin on cardiac structure, function, and circulating biomarkers in patients with type 2 diabetes cardiolink-6 trial. Nephrol Dial Transpl. 2020;35:895-7.

33 Patel RB, Ter Maaten JM, Ferreira JP, McCausland FR, Shan SJ, Rossignol P, et al. Challenges of Cardio-Kidney Composite Outcomes in Large-Scale Clinical Trials. Circulation. 2021 Mar 2;143(9):949-58

34 Ferreira JP, Sharma A, Mehta C, Bakris G, Rossignol P, White WB, et al. Multi-proteomic approach to predict specific cardiovascular events in patients with diabetes and myocardial infarction: findings from the EXAMINE trial. Clin Res Cardiol. 2021 Jul; 110(7):1006-19. 